\title{
Three-dimensional Printing Technology in Surgery
}

\section{Dr. Amit Pate $1^{1^{\star}}$ Dr. Peter Cosman ${ }^{2}$ and Dr. Shashank Desai ${ }^{3}$}

${ }^{1}$ School of Medicine, Griffith University, Queensland, Australia

${ }^{2}$ School of Surgery, University of Western Sydney, Australia

${ }^{3}$ School of Medicine, University of Queensland, Queensland, Australia

\begin{abstract}
Background: Three-dimensional printing has become an increasingly widespread technology, becoming more accessible to individuals, small businesses and organisations. It represents the ability to manufacture objects of various substrate material using computer aided 3D plans. This article aims to explore the various uses of this technology in medicine and surgery including future directions and applications
\end{abstract}

Method: A generalised review of past and current articles was performed exploring the known uses of three-dimensional printing in medicine and surgery.

Results: Broadly, the applications include; education for health professionals and patients, surgical planning and decisionmaking, custom prostheses, tissue scaffolds, and customised surgical instrument manufacturing. Future directions include bionic prostheses, live tissue grafts, and whole organ printing.

Keywords: 3D Printing; Additive manufacturing; Bioprinting

\section{Introduction}

\section{Current concerns regarding the laparoscopic approach}

Three dimensional printing, additive manufacturing or stereolithography as it was first described has recently come into vogue within the scientific community. Initially conceived in the 1980s by Charles Hull, it represents the successive addition of layers of various materials to form high definition three dimensional structures using Cartesian co-ordinates from 3D computer aided design (CAD) software [4,5]. Over the years, advances in biofabrication, have given the ability to use a variety of bio-inert materials, from hydrogels, ceramics and polymers, to bio absorbable glass and even metals $[4,6-8]$.

This technology has been used in a multitude of applications such as architecture, construction, industrial design, automotive engineering and aerospace [8]. This paper explores the application of 3D printing in medicine and surgery, including education, surgical planning, instrument production, tissue scaffolds and prosthetic grafts. Although many are already utilising this technology, its potential has barely been scratched. We believe that in the future, developments in $3 \mathrm{D}$ printing will one day facilitate the printing of graftable tissue and complete organs [8-11]. It is also a technology that if better understood can facilitate further innovation in the field of surgery and medicine.

\section{How it works}

Many software applications; free and otherwise, are available which function as a 3 dimensional canvas for which an object can be designed or transposed. Utilising this software can convert conventional cross sectional imaging such as CT and MRI into blueprints for a computerised three dimensional structure. 3 dimensional printers act to turn the design into a 3D physical structure. It works by sequentially adding layers upon layers of a particular material in order to achieve the three dimensional form. Depending on the size of the structure and the substrate materials the cost of production can be variable. As the technology has been evolving, the costs are plummeting the learning curve is flattening, and accessibility is improving. Depending on size of the size, printing can take anywhere from sub 1 hour to over 20 . With developments in the printing engineering, this is being tightened.

\section{Education}

Historically, anatomical teaching has come by way of cadaveric dissection, studying descriptive text and two-dimensional diagrams. With increasing societal pressures, dwindling cadaveric donations and high overheads in maintaining them, we are being more heavily reliant on 'book learning' which has been proven to lead to suboptimal understanding and poor retention $[1,6,12-14]$. 3D anatomical software aids in being able to manipulate $2 \mathrm{D}$ images giving an improved appreciation for the 3D structure, however it still lacks the tactile element which makes dissection such an ideal learning method [13]. Cast moulded anatomical models started becoming more readily available, which although very useful to learning, can be costly, and not individually customisable to specific design requirements and hence are reliant on what models companies are able to offer. With 3D printing technology, customised models can be printed, using various materials, the cost of which is mainly dependant on the model design, materials, and the initial capital investment required for the printer. These printers are quickly becoming a ubiquitous commodity, and its even possible, to 3D print, the $3 \mathrm{D}$ printers [4,7]. An example of a sturdy, readily available substrate is Acrylonitrile butadiene styrene (ABS), the plastic that also makes Lego blocks. A quick search online It can be bought for consumer price of $\$ 49 \mathrm{AU}$ per $1 \mathrm{~kg}$, which translates to anatomically accurate models of organs and bones for less than a cup of coffee [9]. Unique or complex pathology can also be macroscopically visualised adding real significance to scenario-based learning modules [1] (Figure 1) depicts a printed replica of a prosected hand, exhibiting the vascular and musculotendinous anatomy in great intricacy produced by Monash University [1].

Teaching applications also extend to patients in both pre-operative and post-operative settings. A patient's understanding of his/her particular condition may require some underlying knowledge of anatomy and pathology, which may be provided by means of 3D printed personalised models of the particular organ of interest, thereby promoting a deeper understanding of the condition and surgical procedures that may be considered in management. Such depth of understanding can encourage true autonomous decision making $[1,13,15]$

${ }^{*}$ Corresponding author: Dr. Amit Patel, Lecturer, Griffith University, 56 Maynard Street, Woolloongabba, 4102 QLD, Australia, Tel: +61450116969; E-mail: amitpatel86@ gmail.com

Received November 09, 2015; Accepted December 09, 2015; Published December 14, 2015

Citation: Patel A, Cosman P, Desai S (2015) Three-dimensional Printing Technology in Surgery. Surgery Curr Res 6: 255. doi:10.4172/2161-1076.1000255

Copyright: ( 2015 Patel A, et al. This is an open-access article distributed under the terms of the Creative Commons Attribution License, which permits unrestricted use, distribution, and reproduction in any medium, provided the original author and source are credited. 

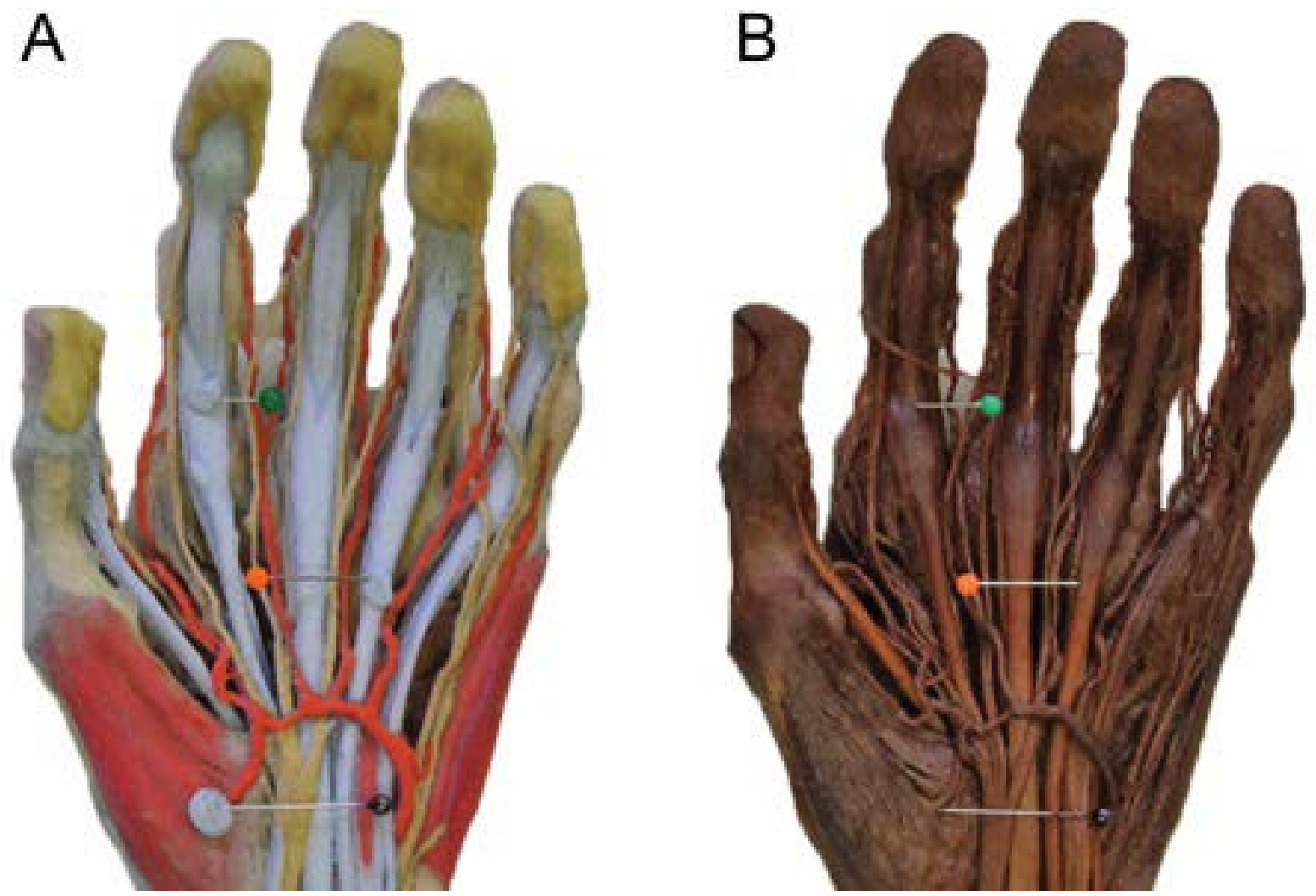

Figure 1: A) Printed replica of a CT scanned original prosected hand shown in B) from the Monash University's Centre for Human Anatomy Education [11].

\section{Surgical planning}

Identifying and visualising anatomy is critical for any surgical intervention, and depending on complexity and variability of the regional anatomy, physical pre-operative anatomical assessment is an important adjunct to surgical planning. Medical imaging has advanced considerably since the development of the roentgenogram, and it is now possible to display three-dimensional anatomy using computerised reconstructions of tomographic and even sonographic imaging modalities. Using these images, a replica or model of the anatomical structures of interest may be created in order to better understand the complexities and potential issues of certain steps of a procedure $[13,16,17]$. This has the potential to reduce complications and unexpected adversities. Such models also add a tactile interface that can amplify the intuitive understanding of anatomy, aiding in novel approaches to unique and complex scenarios. Using life-sized replicas and performing simulations of particular procedures using actual equipment could theoretically increase efficiency and safety $[18,19]$. This technology has already been used in a broad range of scenarios across the board of surgical specialties.

Published examples include model printing for cardiothoracic surgical teams planning a thorascopic subsegmentectomy for lung cancer [18], and the surgical correction of complex congenital anomalies such as subpulmonary ventricular septal defects [20]. Recently the Urological team at the Tulane University School of Medicine in New Orleans presented 5 patients where 3D models of renal tumours were printed for decision-making for nephronsparing partial nephrectomies for renal tumours, resulting in successful partial resections with clear margins [21]. Quintini et al., have used semitransparent printed models for planning liver transplantation, allowing familiarisation of normally occluded critical structures allowing a more intuitive understanding of the individual patient's anatomy during surgery [22]. Neurosurgical teams have also described pre-operative simulation surgery using $3 \mathrm{D}$ printed models using materials of similar characteristics as brain tissue incorporating deep brain tumours [17]. Similarly, models of cerebral vasculature have been have been used for pre-operative planning and training for cerebral aneurysm clippings. An example is depicted in Figure 2. The model design also includes replaceable aneurysmal segments to allow for repeated use [2,17,19 ]. In Maxillofacial surgery, mandibular reconstructions have been significantly aided by the ability to print pre and planned post-resection models, to allow fitted bone plates to be fabricated, and for bone grafts to be accurately cut for superior healing and aesthesis using sterile 3D printed cutting guides [23-25].

\section{Surgical equipment and devices}

Hundreds of surgical instruments exist in the surgeons' repertoire; each specifically designed to serve a very specific function. Advances in technique occasionally necessitate production of novel instruments. Traditionally, production of custom equipment requires a very large capital input. Because of this, some tools may not be financially viable, especially if their use is reserved for rare, novel or patient-specific procedures. The advantage of $3 \mathrm{D}$ printing, is that production is dependent on only few key considerations: An appropriate design, appropriate material, availability of technology that allows "printing" of such material and suitable sterilisation techniques. The interplay between these factors allows for variability of designs to cater for specific needs.

Polylactic acid (PLA) is a biodegradable thermoplastic polymer used in 


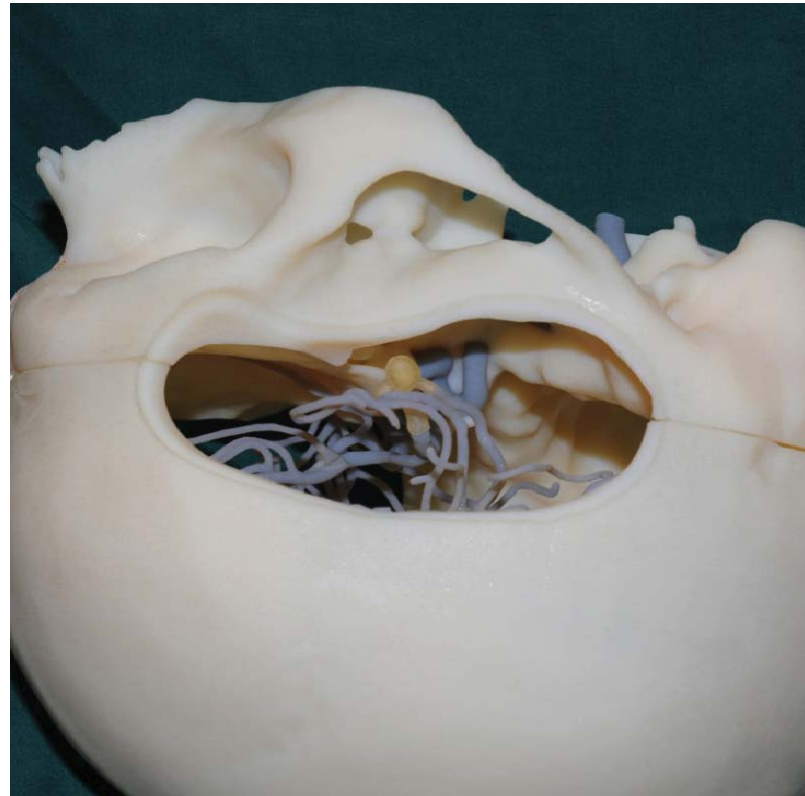

Figure 2: A) Model of cranium with embedded micro-vascular anatomy facilitating microsurgical simulation and planning for aneurysm clipping.

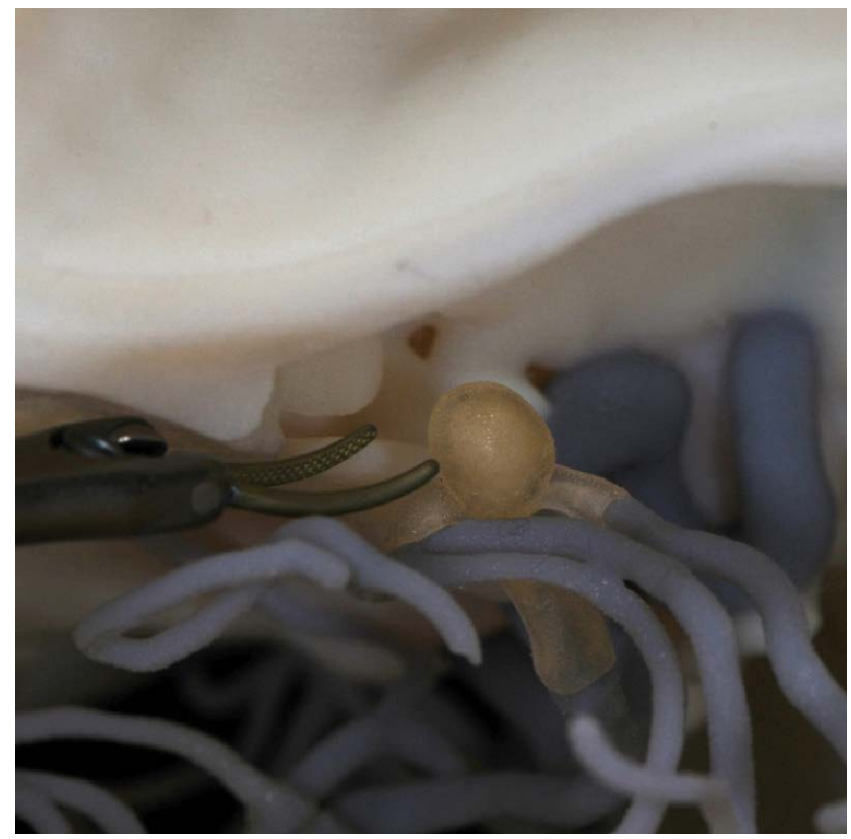

Figure 2: B) Replaceable aneurysmal segment enabling repeated use of the model [21].

many applications. It is the second most consumed bioplastic in the world due to being relatively inexpensive, readily available, biodegradable, and with the right design and structure - durable with a high melting point. These physiochemical characteristics make it an ideal substrate for 3D printed instruments $[6,8]$. One recent study produced on-demand 3D printed PLA basic surgical set for long-duration space missions. The researchers managed to reproduce printable versions of a basic laparotomy instrument set deemed functionally adequate by a panel of surgeons [26]. Similarly Kondor et al. explored the possibility of a 3D-printable military trauma toolset, and demonstrated its successful use on a simulation model, with favourable results [27]. Rankin et al. conducted a feasibility study for a 3D-printed retractor confirming adequate tensile strength, low cost, and immediate sterility post printing allowing immediate use [28].

Increased availability of and access to, open-source coding, is making 3D design and modelling much more feasible, with a learning curve for design and production that is beginning to plateau. This not only allows replication of existing instruments, but also facilitates engineering de novo instruments for novel purposes. For example, the University of Illinois Plastics and Reconstructive Surgical team successfully developed a custom bone reduction clamp using 3D printing technology, in order to gain multi-point reduction in small long bone fractures of the hand. The design incorporates specific grooves to act as support guides for K-wire insertion at a fraction of the price of commercially manufactured custom instruments [29]. Pelvic neoplasms are notorious for poor prognosis, and this is largely due to difficulty in achieving clear resection margins. The complexity of the pelvic anatomy and the confined space bounded by the bony pelvis makes surgery very difficult. Previous simulation studies have shown promising results with patient-specific instruments for this purpose [30]. Recently the Orthopaedic unit at Sir Charles Gairdner Hospital in Nedlands developed a customised osteotomy guide for resection of a chondrosarcoma of the pubic ramus. Planned resection planes were mapped onto the model, and using computer design software a 'cutting block' osteotomy guide tool was designed to aid in accurate resection of the osteosarcoma with margins clear of tumour [31].

\section{Prosthetics and bionics}

Prostheses have been available for many years, but only recently are we fabricating theses prostheses specific to the patient. For years, implants of various sizes have been mass-produced for off-the shelf selection of a size suitable for the given situation. Modern printing technology has allowed patient specific customised prosthetic devices to be manufactured, which could mean improved patient outcomes by way of improved aesthetic results, functional outcome and prosthesis stability. Design can be customised in order promote tissue incorporation. For example the porous design of bone prostheses using hydroxyapatite or inert metals such as titanium encourages osteogenesis and better incorporation into natural tissues [32]. An example is the use of these materials in prostheses of the cranial vault, resulting in aesthetically pleasing symmetrical reconstruction with satisfactory bioincorporation for longer term stability [33-37].

Custom pre-contoured bone plates for maxillofacial surgery have been well described which can aid in precise bone graft harvesting and can also reduce operative times by rationalising the contouring process $[9,38]$.

Various case-specific prostheses have been in use in orthopaedic surgery. Multiple studies have explored the use of custom prostheses to facilitate limb sparing tumour resection and reconstruction in Hemipelvectomy surgery. Although prognosis remains poor oncologically, the implant can provide a functionally superior outcome to those with full limb amputations. Most studies are small in number and utilise varying reconstruction methods, which make it difficult to draw definitive conclusions on efficacy [39,40]. A recent example is shown in Figure 3, designed and implanted by Gerrard et al. in a lady requiring a hemipelvectomy for osteosarcoma. They were able to create a porous implant with a medialised acetabular facet, an anterior plate to secure to the pubic ramis, and a guide for bolt placement through the sacrum into the contralateral ilium. It has allowed the patient to retain her native leg, and mobilise with minimal extrinsic support [3].

Open-source projects are becoming increasingly popular, giving patients the power to even print their own prostheses. Groups such as 'Enabling the Future' have dedicated their efforts towards accessible solutions to upper limb 


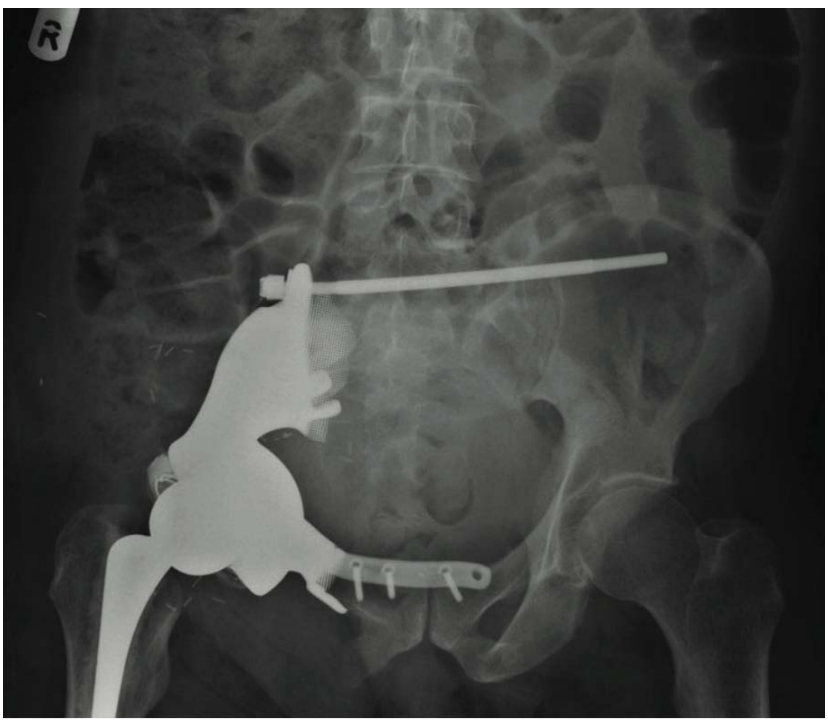

Figure 3: Post-operative roentograph of an implanted hemipelvectomy prosthesis [40]

amputations [41]. Examples of these devices include the 'Robohand' and "Cyborg beast". They are extrinsic 3D printed non-invasive, external braces that utilise existing muscular movement to replicate the function of joints, fingers and hands. Depending on the specific case, the results have been shown to be comparable to the much more costly myoelectric prosthetic alternatives [42]. Myoelectric prostheses are incredible pieces of technology, however, their cost severely restrict widespread use, especially in poorer countries where access and means are restricted [43].

\section{Scaffolding and biosynthetic grafts}

Neoplasia, trauma, infection, ischaemia, and congenital disease can all result in growth abnormalities, and structural anomalies requiring extrinsic scaffolding or grafts for tissue regeneration. The ideal substrate is autologous tissue, however in many situations the supply cannot match the demand. A tissue scaffold must be biocompatible, allow cellular incorporation, promote adherence, foster growth, retain structure via suitable crosslinking mechanisms, and have a micro-architecture to facilitate blood flow and tissue vitality. Investigation into the utility of various suitable materials is well underway.

Studies have shown that 3D-printed porous scaffolds using combinations of calcium phosphate ceramics, hydroxyapatite, calcium phosphate cements, monetite, brushite, and even inert metals such as titanium can be used as effective bone-mimicking scaffolds to allow autologous bone incorporation, either strengthening a metallic prosthesis, or allowing bioabsorption of the mineral scaffold to be replaced by autologous bone. Newer materials have also been developed such as B-TCP, bioactive glass A and B mixtures which stimulate bone migration, growth and can differentially incorporate certain soft tissues [11,32,44,45]. Soft tissues also require a porous biocompatible substrate to encourage cellular migration and viability. Soft tissue manufacture has leapt forward with the ability to print various hydrogels. This technology has a very wide application range, a few examples are discussed here. Recently a bioreabsorbable patient specific 3D printed tracheal airway splint was created from polycaprolactone and successfully inserted in an infant with severe airway occlusion from tracheobronchomalacia. Post operative recovery was satisfactory and absorption of the construct is estimated to be circa 3 years [46]. Another study has shown promising results in producing graftable cartilaginous constructs using infrapatellar bursa derived adipose stem cells embedded in 3D printed chitosan scaffolds. This can change the way we manage osteoarthritis and will aid in producing multiple cell type tissue complexes in the future [47]. Neuronal injury remains a clinical conundrum. Slow regeneration and healing make it difficult for neuronal incorporation into scaffolds and grafts. Recent studies show the use of micro-fibre electrospinning to fabricate nerve-guiding scaffolds mimicking epineurium and perineurium for aided axonal regeneration. A segment of the mouse common fibular nerve has been successfully replaced with a graft using this method, showing regeneration rates similar to that of autologous nerve grafting $[48,49]$. This has great potential for nerve repair in particularly in the trauma setting. Another example is the use of $3 \mathrm{D}$ printed type 1 collagen hydrogel scaffolds for the fabrication of patient specific auricles for patients suffering from microtia with promising results in recent animal studies. The collagen hydrogel promotes donor chondrocyte seeding in order to produce tissue of appropriate cellular density, size and structure. This has shown to mimic normal biomechanical properties better than autologous reconstructions and avoid undesirable shape distortion and shrinkage $[50,51]$.

Although in its infancy, bionic implants are soon to become a reality. Using cartilaginous hydrogels incorporating silicon support, with embedded silver nanofibre technology, Manoor et al. at Princeton University have grown a morphologically correct implantable external ear capable of detecting and conducting sound. This has proven to be a successful proof of concept for the printable integration of tissues and electronics [52].

Scaffolds also have a role ex-vivo. A large impediment to drug development and safety testing has been the lack of reproducible tissue adequately similar to natural human tissue. Cell cultures, and genomically similar animal models are currently being used to attempt to mimic human biophysiology and pharmacodynamics. Unfortunately, the cellular interplay between in-vivo cells are also dependant on its microenvironment, including molecular signalling, and extracellular matrix attachment. Using 3D printed rigid lattices of carbohydrate-glass as a cytocompatible sacrificial scaffold for engineered cellular tissue, we are able to seed human embryonic vascular endothelial cells (HUVECs) within the formed microchannels, essentially forming a vascular network, theoretically allowing a way to ensure tissue perfusion, and maintain a healthy block of cultured tissue. This has been demonstrated and has been successful in rat hepatocyte cultures [44]. Hooper et al., have used this technology to create endothelial microchannels to mimic vessels within breast tissue, which has allowed differential breast tumour transendothelial migration to be demonstrated based on molecular signalling between tumour cells and endothelium demonstrating the importance of biomolecular signalling in invasiveness and metastatic potential of particular tumours [53]. This in-vitro model may also have a future role in pharmological trials for chemotherapeutics.

\section{Future directions}

Paradoxically, although use of this technology will currently only be accessible to those working in financially well equipped institutes and countries, 3D printing has a potential role in those short of resource. With an easily conceivable library of 3D designs - an array of educationally invaluable anatomical models, surgical trainers and instruments could be produced for medical training facilities in the developing world. On demand sets utilising low cost substrates, can potentially result in eco-friendly economical surgical practice. Currently an important consideration is the printing time needed. Although theoretically sound, the time it takes to print "on-demand" sets is not feasible in an acute setting. With improving printer technology, this is quickly improving, and no doubt will become a reality in the near future. 
3D printing alongside the rapidly advancing field of tissue engineering is quickly expanding the horizon of whats going to be possible in the future.

Currently around the globe, 3D printed prostheses are already being used. A few examples include; Cranial bone patches, Facial bone replacements, Joint replacements, and large vessel grafts.

In animal trials at the moment are synthetic nerve grafts - which hope to replace the use of autologous nerve grafts that incur donor site morbidity. If successful will have a considerable impact on approaches to trauma and reconstruction surgery and could improve patients' functional outcome.

As we gain confidence in culturing stem cells and utilising their pluripotential for forming well-diversified functional tissues, we get closer to creating tissues similar to our own. Clinically, this means having human tissue samples in a form that better exhibits features of in-vivo tissue. 3D Printing allows will be the bridge to enabling this advancement. It allows us to form the scaffold essential for vitality of these tissues as well as encourage structure and form as close as possible to This will be useful for pharmaceutical development and safety testing. It also opens the door for a myriad of further research into understanding our own cellular physiology. It will reduce animal testing making scientific testing less ethically ambiguous.

The cruel duality of the human body is that its complexity and efficiency of our various organs is counterbalanced by irreplaceability, and vulnerability. Unfortunately many diseases prevent the body from fully recovering premorbid function, some of these functions vital for life. Transplantation medicine has provided an invaluable way to replace defective organs. Unfortunately graft rejection reactions; immunosuppression related morbidity and lack of adequate supply preclude its use for many patients. Perhaps the most anticipated use for the three dimensional printing in Medicine is organ printing. Though we are many years from a complete functional organ replacement, it is scientifically feasible and the direction of much research. Once we achieve this feat, it will have a massive implication for medicine and surgery.

\section{Conclusion}

Three-dimensional printing is a highly customisable technology, which is changing the way we approach problems in medicine and surgery. It can be used as an adjunct to anatomical education - for health professionals, students, and patients alike. It is helping plan surgical procedures, customise surgical tools, and fabricate patient specific prostheses. It is actively being used for forming high definition tissue moulds and scaffolds, which in conjunction with tissue engineering, are paving the way towards a future where we are able to print functional organs using patients' own cells. It is a useful tool to facilitate innovative ideas, create novel instruments, and if geared in a correct direction, can be used for the greater good on a global scale. Although we are still far away from this goal, three-dimensional printing has the potential to significantly improve medical and surgical practice and we implore clinicians to think outside the box and consider the capabilities of the technology when looking to find new approaches to teaching and clinical practice.

\section{Acknowledgements}

Dr Bhaveshkumar Patel, FRACS, MBChB, Senior Lecturer (University of Queensland - School of Medicine)

\section{References}

1. McMenamin PG, Quayle MR, McHenry CR, Adams JW (2014) The production of anatomical teaching resources using three-dimensional (3D) printing technology. Anat Sci Educ 7: 479-486.

2. Wurm G, Lehner M, Tomancok B, Kleiser R, Nussbaumer K (2011) Cerebrovascular biomodeling for aneurysm surgery: simulation-based training by means of rapid prototyping technologies. Surg Innov 18: 294-306.

3. Gerrard C (2014) 3D Printing: A Clinicians Experience. Ann R Coll Surg Eng 96: $230-231$

4. Horvath J (2014) A Brief History of 3D Printing. Mastering 3D Prtinting. Apress; p. 3-10.

5. Chang PS, Parker TH, Patrick CW Jr, Miller MJ (2003) The accuracy of stereolithography in planning craniofacial bone replacement. J Craniofac Surg 14: $164-170$.

6. Rengier $F$, Mehndiratta A, Von Tengg-Kobligk $H$, Zechmann CM, Unterhinninghofen R, et al. (2010) 3D printing based on imaging data: Review of medical applications. International Journal of Computer Assisted Radiology and Surgery 5: 335-341.

7. Gross BC, Erkal JL, Lockwood SY, Chen C, Spence DM (2014) Evaluation of $3 D$ printing and its potential impact on biotechnology and the chemical sciences. Anal Chem 86: 3240-3253.

8. Lipson H, Kurman M (2013) Fabricated: The New World of 3D Printing. John Wiley \& Sons, Inc., Indianapolis, Indiana.. $320 \mathrm{p}$

9. Gerstle TL, Ibrahim AM, Kim PS, Lee BT, Lin SJ (2014) A plastic surgery application in evolution: three-dimensional printing. Plast Reconstr Surg 133 446-451.

10. Huang W, Zhang X1 (2014) 3D Printing: Print the future of ophthalmology. Invest Ophthalmol Vis Sci 55: 5380-5381.

11. Murphy SV, Atala A1 (2014) 3D bioprinting of tissues and organs. Nat Biotechno 32: $773-785$.

12. Shiraishi I, Kurosaki K, Kanzaki S, Ichikawa H (2014)Development of Suep Flexible Replica of Congenital Heart Disease with Stereolithography 3D Printing for Simulation Surgery and Medical Education. J Card Fail 20: 180-181.

13. Preece D, Williams SB, Lam R, Weller R (2013) “Let's get physical”: advantages of a physical model over 3D computer models and textbooks in learning imaging anatomy. Anat Sci Educ 6: 216-224.

14. Nibblett B, Marie DVM Mvs, Dascanio J, Bauman E, PhD RN, Pederson D, et al. (2013)Board 527 - Technology Innovations Abstract The Use of 3D Printing to Generate Teaching Models in Veterinary Medicine (Submission \#985). Simul Healthc.;8(6):622.

15. Schmauss D, Haeberle S, Hagl C, Sodian R (2015) Three-dimensional printing in cardiac surgery and interventional cardiology: a single-centre experience. Eur J Cardiothorac Surg 47: 1044-1052.

16. Esses SJ, Berman P, Bloom Al, Sosna J (2011) Clinical applications of physical 3D models derived from MDCT data and created by rapid prototyping. AJR Am J Roentgenol 196: W683-688.

17. Oishi M, Fukuda M, Yajima N, Yoshida K, Takahashi M, et al. (2013) Interactive presurgical simulation applying advanced $3 \mathrm{D}$ imaging and modeling techniques for skull base and deep tumors. J Neurosurg 119: 94-105.

18. Nakada T, Akiba T, Inagaki T, Morikawa T(2014) Thoracoscopic anatomica subsegmentectomy of the right S2b + S3 using a 3D printing model with rapid prototyping. Interact Cardiovasc Thorac Surg 19: 696-698.

19. Kimura T, Morita A, Nishimura K, Aiyama H, Itoh H, et al.(2009) Simulation of and training for cerebral aneurysm clipping with 3-dimensional models. Neurosurgery 65: 719-726.

20. Sodian R, Weber S, Markert M, Rassoulian D, Kaczmarek I, et al. (2007) Stereolithographic models for surgical planning in congenital heart surgery. Ann Thorac Surg 83: 1854-1857.

21. Silberstein JL, Maddox MM, Dorsey P, Feibus A, Thomas R, et al. (2014 Physical models of renal malignancies using standard cross-sectional imaging and 3-dimensional printers: A pilot study. Urology 84: 268-272.

22. Quintini C, Aucejo F, Hashimoto K, Zein N, Miller C (2014)State of the Art and Future Developments for Surgical Planning in LDLT. Curr Transplant Reports 1: $35-42$.

23. Cohen A, Laviv A, Berman P, Nashef $R$, Abu-Tair J (2009) Mandibula reconstruction using stereolithographic 3-dimensional printing modeling technology. Oral Surg Oral Med Oral Pathol Oral Radiol Endod 108: 661-666.

24. Zenha H, Azevedo L, Rios L, Pinto A, Luz Barroso M, et al. (2011)The application of 3-D biomodeling technology in complex mandibular reconstructionexperience of 47 clinical cases. Eur J Plast Surg 34: 257-265. 
Citation: Patel A, Cosman P, Desai S (2015) Three-dimensional Printing Technology in Surgery. Surgery Curr Res 6: 255. doi:10.4172/21611076.1000255

25. Logan H, Wolfaardt J, Boulanger P, Hodgetts B, Seikaly H (2013) Exploratory benchtop study evaluating the use of surgical design and simulation in fibula free flap mandibular reconstruction. J Otolaryngol Head Neck Surg 42: 42.

26. Wong JY, Pfahnl AC (2014) 3D printing of surgical instruments for long-duration space missions. Aviat Space Environ Med 85: 758-763.

27. Kondor S, Grant G, Liacouras P, Schmid JR, Parsons M, et al. (2013) On Demand Additive Manufacturing of a Basic Surgical Kit. J Med Device 7: 030916-030911.

28. Rankin TM, Giovinco NA, Cucher DJ, Watts G, Hurwitz B, et al. (2014) Threedimensional printing surgical instruments: are we there yet? J Surg Res 189 193-197.

29. Fuller SM, Butz DR, Vevang CB, Makhlouf MV2 (2014) Application of 3-dimensional printing in hand surgery for production of a novel bone reduction clamp. J Hand Surg Am 39: 1840-1845.

30. Cartiaux O, Paul L, Francq BG, Banse X, Docquier PL. Improved accuracy with 3D planning and patient-specific instruments during simulated pelvic bone tumor surgery. Annals of Biomedical Engineering. 2014. p. 205-13.

31. Blakeney WG, Day R, Cusick L, Smith RL (2014) Custom osteotomy guides for resection of a pelvic chondrosarcoma. Acta Orthop 85: 438-441.

32. Sidambe A (2014) Biocompatibility of Advanced Manufactured Titanium Implants-A Review. Materials (Basel) 7: 8168-8188.

33. Staffa G, Nataloni A, Compagnone C, Servadei F (2007) Custom made cranioplasty prostheses in porous hydroxy-apatite using 3D design techniques: 7 years experience in 25 patients. Acta Neurochir (Wien) 149: 161-170.

34. Eppley BL1 (2002) Craniofacial reconstruction with computer-generated HTR patient-matched implants: use in primary bony tumor excision. J Craniofac Surg 13: 650-657.

35. Kim BJ, Hong KS, Park KJ, Park DH, Chung YG, et al. (2012) Customized cranioplasty implants using three-dimensional printers and polymethylmethacrylate casting. J Korean Neurosurg Soc 52: 541-546

36. Miles BA, Sinn DP, Gion GG (2006) Experience with cranial implant-based prosthetic reconstruction. J Craniofac Surg 17: 889-897.

37. Mulliken GH, Bichot NP2, Ghadooshahy A2, Sharma J3, Kornblith S4, et al (2015) Custom-fit radiolucent cranial implants for neurophysiological recording and stimulation. J Neurosci Methods 241: 146-154.

38. Mayrink G, Asprino L, William R, Moreira F, Henrique G, Paschoal DL, et al.(2011) Using biomodels for maxillofacial surgeries?: 10 years of experience in a Brazilian public service. Brazialian J Oral Sci 10: 294-296.

39. Sun W, Li J, Li Q, Li G, Cai Z (2011) Clinical Effectiveness of Hemipelvic Reconstruction Using Computer-Aided Custom-Made Prostheses After
Resection of Malignant Pelvic Tumors. J Arthroplasty [Internet]. Elsevier Inc 26: 1508-1513.

40. Mayerson JL, Wooldridge AN, Scharschmidt TJ (2014) Pelvic resection: current concepts. J Am Acad Orthop Surg 22: 214-222.

41. Enabling The Future | A Global Network Of Passionate Volunteers Using 3D Printing To Give The World A "Helping Hand." [Internet]. [cited 2015 Apr 4]

42. Robohand? Customised, Fitted, Mechanical Fingers and Hands [Internet] [cited 2015 Feb 15]. Available from: http://www.robohand.net/

43. Zuniga J, Katsavelis D, Peck J, Stollberg J, Petrykowski M, et al. (2015) Cyborg beast: a low-cost 3d-printed prosthetic hand for children with upperlimb differences. BMC Res Notes 8: 10.

44. Miller JS, Stevens KR, Yang MT, Baker BM, Nguyen D-HT, et al. (2012) Rapid casting of patterned vascular networks for perfusable engineered threedimensional tissues. Nat Mater 11: 768-774.

45. Bose S, Vahabzadeh S, Bandyopadhyay A (2013) Bone tissue engineering using 3D printing. Mater Today 16: 496-504.

46. Zopf DA, Hollister SJ, Nelson ME, Ohye RG, Green GE (2013) Bioresorbable airway splint created with a three-dimensional printer. N Engl J Med 368: 20432045.

47. Ye K, Felimban R, Traianedes K, Moulton SE, Wallace GG, et al. (2014) Chondrogenesis of infrapatellar fat pad derived adipose stem cells in 3D printed chitosan scaffold. PLoS One 9: e99410.

48. Pateman CJ, Plenderleith R, Daud M, Harding A, Christmas C, et al. (2013) 3D Micro-Printing of Nerve Guides for Peripheral Nerve Repair. Eur Cells Mater 26: 78.

49. Kolar M, Pateman C, Novikov (2014) P-74: Comparison of 3D Micro-Printed Polycaprolactone Conduits and Fibrin Conduits for Peripheral Nerve Repair Tissue Cell Eng Soc Conf 23(Supplement)

50. Reiffel AJ, Kafka C, Hernandez KA, Popa S, Perez JL, et al. (2013) High-fidelity tissue engineering of patient-specific auricles for reconstruction of pediatric microtia and other auricular deformities. PLoS One 8: e56506.

51. Rankin TM, Mailey B, Cucher D, Giovinco NA, Armstrong DG, et al. (2014) Use of $3 \mathrm{D}$ Printing for Auricular Template Molds in First Stage Microtia Traumatic Optic Neuropathy. Plast Surg 134: 16-17.

52. Mannoor MS, Jiang Z, James T, Kong YL, Malatesta KA, et al. (2013) 3D printed bionic ears. Nano Lett 13: 2634-2639.

53. Hooper RC, Karina A, Joyce J, Jacoby A, Asanbe O, et al. (2014) A Novel 3D Platform to Investigate Neoangiogenesis, Transendothelial Migration and Metastasis of Breast Cancer Cells. Plast Reconstr Surg 133: 169. + 